\title{
Phytoprotection
}

\section{État actuel de la lutte biologique contre les acariens phytophages par des acariens prédateurs}

\section{Roy}

Volume 74, numéro 1, 1993

URI : https://id.erudit.org/iderudit/706035ar

DOI : https://doi.org/10.7202/706035ar

Aller au sommaire du numéro

Éditeur(s)

Société de protection des plantes du Québec (SPPQ)l

ISSN

0031-9511 (imprimé)

1710-1603 (numérique)

Découvrir la revue

Citer cet article

Roy, M. (1993). État actuel de la lutte biologique contre les acariens phytophages par des acariens prédateurs. Phytoprotection, 74(1), 41-49. https://doi.org/10.7202/706035ar
Résumé de l'article

Au Québec, la stratégie de lutte contre le tétranyque rouge du pommier (Panonychus ulmi) et le tétranyque à deux points (Tetranychus urticae) dans les vergers de pommier (Malus pumila) repose actuellement sur l'emploi d'acaricides coûteux. Par ailleurs, les consommateurs exigent de plus en plus des fruits exempts de pesticides. Dans ce contexte, l'utilisation de méthodes de lutte non chimiques suscite un grand intérêt auprès des pomiculteurs. Depuis plusieurs années, les efforts de recherche et les essais conduits aux États-Unis et en Europe ont donné un large essor à l'utilisation de souches d'acariens prédateurs résistants à certaines classes de pesticides. Au Québec, des expériences de lutte biologique avec l'acarien prédateur Amblyseius fallacis [Acarina: Phytoseiidae] ont été entreprises depuis quelques années. Cet article se propose d'exposer la problématique de la lutte contre les acariens nuisibles dans les vergers québécois ainsi que les perspectives d'avenir de l'utilisation de la lutte biologique contre ces ravageurs. 


\title{
État actuel de la lutte biologique contre les acariens phytophages par des acariens prédateurs
}

\author{
Michèle Roy ${ }^{1}$
}

Reçu 1992-11-18; accepté 1993-05-11

Au Québec, la stratégie de lutte contre le tétranyque rouge du pommier (Panonychus ulmi) et le tétranyque à deux points (Tetranychus urticae) dans les vergers de pommier (Malus pumila) repose actuellement sur l'emploi d'acaricides coûteux. Par ailleurs, les consommateurs exigent de plus en plus des fruits exempts de pesticides. Dans ce contexte, l'utilisation de méthodes de lutte non chimiques suscite un grand intérêt auprès des pomiculteurs. Depuis plusieurs années, les efforts de recherche et les essais conduits aux États-Unis et en Europe ont donné un large essor à I'utilisation de souches d'acariens prédateurs résistants à certaines classes de pesticides. Au Québec, des expériences de lutte biologique avec l'acarien prédateur Amblyseius fallacis [Acarina: Phytoseiidae] ont été entreprises depuis quelques années. Cet article se propose d'exposer la problématique de la lutte contre les acariens nuisibles dans les vergers québécois ainsi que les perspectives d'avenir de l'utilisation de la lutte biologique contre ces ravageurs.

Roy, M. 1993. Current status of biological control against phytophagous mites by predaceous mites. PHYTOPROTECTION 74: 41-49.

Québec's control strategy against mite pests such as Tetranychus urticae and Panonychus ulmi in apple (Malus pumila) orchards relies currently on the use of expensive miticides. However consumers demand pesticide-free fruits. As a result, apple producers are interested in the use of non-chemical control methods. American and european research efforts conducted over many years lead to the increased use of predaceous mite strains resistant to certain categories of pesticides. Biological control experiments conducted in Québec over the past few years focused on the predaceous mite Amblyseus fallacis [Acarina: Phytoseiidae]. This paper presents the intricate problem of noxious mite control in Québec apple orchards, as well as future research avenues for the use of biological control against these pests.

\section{INTRODUCTION}

Au Québec, deux espèces de tétranyques sont considérées comme des ravageurs prépondérants dans les vergers de pommiers (Malus pumila Mill.) (Roy et Vincent 1988). Ce sont le tétranyque rouge du pommier, Panonychus ulmi (Koch) [Acarina: Tetranychidae] et le tétranyque à deux points, Tetranychus urticae Koch [Acarina: Tetranychidae]. Actuellement, les pomiculteurs doivent intervenir à raison d'un à trois traitements acaricides au cours de la saison pour les réprimer (Savoie et al. 1992) ${ }^{2}$. En 1991, pour les vergers pilotes du Réseau d'avertissements phytosanitaires du ministère de

1. Service de phytotechnique de Québec, ministère de l'Agriculture, des Pêcheries et de l'Alimentation du Québec, 2700, rue Einstein, Sainte-Foy (Québec) Canada G1P 3W8

2. Savoie, L., D. de Oliveira et M. Roy. 1992. Enquête sur les pratiques phytosanitaires des pomiculteurs québécois en 1990. Rapport présenté à la Direction de la recherche et du développement du MAPAQ. 46 pp. 
l'Agriculture, des Pêcheries et de l'Alimentation du Québec (M.A.P.A.Q.), les acaricides ont représenté jusqu'à $30 \%$ du coût total des pesticides utilisés (Roy et Laplante 1992) ${ }^{3}$. On rapporte de plus en plus de problèmes liés à l'efficacité même des acaricides. Toutefois aucun organisme n'a entrepris d'étude pour démontrer le développement de souches de tétranyques résistantes. À cette problématique s'ajoute la préoccupation grandissante des consommateurs québécois face à I'utilisation des pesticides (Anonyme 1991) ${ }^{4}$.

Dans ce contexte, l'utilisation de méthodes de lutte biologique présente un grand intérêt pour les pomiculteurs québécois, d'autant plus que depuis une vingtaine d'années, les efforts de recherche et les essais conduits dans plusieurs pays ont permis une lutte efficace contre les acariens phytophages avec des souches d'acariens prédateurs de la famille des Phytoséiides. Au Québec, des projets de lutte biologique ont été entrepris depuis peu et les efforts de recherche se poursuivent (Bostanian et Coulombe 1986; M.A.P.A.Q. 1992).

L'objet de ce travail est de faire le point sur le recours à la lutte biologique pour réprimer les tétranyques nuisibles dans les vergers québécois. D'abord, on y décrira les principales espèces $d^{\prime}$ acariens nuisibles, leur importance ainsi que les moyens de lutte utilisés pour les réprimer. Ensuite, on y présentera le développement de la lutte biologique dans le monde ainsi que les essais récents de lutte biologique au Québec contre ces acariens pour enfin discuter des perspectives d'avenir de l'utilisation des acariens prédateurs en vergers québécois.

3. Roy, M. et G. Laplante. 1992. Protection des vergers (Réseau-pommier). Rapport d'activités 1991. Ministère de l'Agriculture, des Pêcheries et de l'Alimentation du Québec. Saint-Hyacinthe, Québec. $136 \mathrm{pp}$

4. Anonyme. 1991. Projet pour une stratégie phytosanitaire. Document de consultation. Ministère de l'Agriculture, des Pêcheries et de I'alimentation du Québec et Union des producteurs agricoles. $87 \mathrm{pp}$.

\section{ÉTAT DE LA SITUATION}

Au Québec, comme dans le nord-est américain et dans plusieurs pays européens, le tétranyque rouge du pommier est considéré comme un ravageur important depuis l'introduction du DDT, il y a plus de 40 ans (Parent et Beaulieu 1957). Cet acarien phytophage, introduit d'Europe, hiberne au stade d'oeuf sur l'écorce, les branches ou le tronc des pommiers. L'éclosion des oeufs arrive vers le stade phénologique du pré-bouton rose sur le pommier. Les jeunes tétranyques se dirigent alors vers les feuilles pour se nourrir. Sous nos conditions, on compte de six à huit générations de cet acarien qui se chevauchent pendant l'été. Les populations atteignent leurs densités maximales vers la fin du mois de juillet ou au début du mois d'août (Parent et Pilon 1978).

Le tétranyque à deux points hiberne sous forme de femelles adultes surtout dans les paillis des plantes de couverture du verger ainsi que sous les écorces et dans les fentes des troncs d'arbres. Au Québec, l'espèce complète de cinq à six générations par année (Paradis 1955). Jusqu'à la fin des années 70 , le tétranyque à deux points a été considéré comme un ravageur secondaire en vergers de pommiers. Depuis une dizaine d'années, les populations de cet acarien ont souvent atteint des niveaux élevés dans les plantations de pommiers nains et semi-nains (Roy et Vincent 1989; Vincent et al. 1990). D'après certaines études américaines (Flexner et al. 1991; Hislop et Prokopy 1981; Pfeiffer 1986; Rock et Yeargan 1973), I'utilisation d'herbicides, une pratique courante dans ce type de verger, serait responsable de ces infestations. En effet, la destruction du couvert végétal provoquerait la migration massive des tétranyques à deux points sur les arbres et certains herbicides comme le glyphosate [ $N$-(phosphonomethyl) glycine] (Hislop et Prokopy 1981) et le paraquat $\left(1,1^{\prime}\right.$-dimethyl-4,4'-bipyridinium dichloride) (Pfeiffer 1986) causeraient en plus une mortalité élevée chez les populations d'acariens prédateurs.

D'après Parent et Pilon (1978), une dizaine d'espèces d'arachnides et d'acariens se nourrissent des oeufs et des 
stades immatures du P. ulmi. Les principaux insectes prédateurs de tétranyques sont des thrips, des coccinelles, des pentatomides, des anthocorides, des mirides et des chrysopes. La mortalité des stades immatures de tétranyque rouge varie de 44 à $80 \%$. La prédation représente donc un des principaux facteurs de mortalité. Selon Parent (1973), les coccinelles sont très actives à l'automne sur les oeufs d'hiver du tétranyque rouge. Certaines espèces comme le Coccinella trifasciata perplexa Mulsant [Coleoptera: Coccinellidae], le C. transversoguttata Faldermann [Coleoptera: Coccinellidae], et l' Hippodamia tredecimpunctata tibialis Say [Coleoptera: Coccinellidae], sont plus abondantes mais le Stethorus punctum Leconte [Coleoptera: Coccinellidae], et $S$. punctillum Weise [Coleoptera: Coccinellidae], seraient plus efficaces. Parent et Pilon (1978) ont observé plusieurs espèces d'acariens prédateurs soit: Agistemus fleschneri Summers [Acarina: Stigmaeidae], Allothrombium lerouxi Moss [Acarina: Trombidiidae], Anystis agilis Banks [Acarina: Anystidae], Balaustium sp. [Acarina: Erythraeidae], Amblyseius fallacis (Garman) [Acarina: Phytoseiidae], Typhlodromus longipilus Nesbitt [Acarina: Phytoseiidae], Typhlodromus pomi (Parrot) [Acarina: Phytoseiidae], Typhlodromus soleiger (Ribaga) [Acarina: Phytoseiidae], Typhlodromus rhenanus Oudemans [Acarina: Phytoseiidae], Phytoseius spoofi Oudemans [Acarina: Phytoseiidae], et Zetzellia mali (Ewing) [Acarina: Stigmaeidae]. Selon Paradis (1955), les principaux ennemis naturels du tétranyque à deux points sont l'Amblyseius fallacis, I'Aplothrips faurei Ed. [Thysanoptera: Thripidae] et I'Adalia bipunctata (L.) [Coleoptera: Coccinellidae]. D'après Parent (1967), le $T$. rhenanus s'attaque particulièrement au tétranyque à deux points et le $Z$. mali préfère le tétranyque rouge du pommier. Parent et Pilon (1978) ont établi une relation étroite entre les pullulations du tétranyque rouge du pommier et l'absence de prédateurs dans les vergers ayant été traités avec des insecticides.

Depuis l'implantation des vergers à haute densité de pommiers au début des années 80 , aucune étude n'a porté sur le rôle et la diversité des ennemis naturels d'acariens phytophages présents dans ce type de plantation. D'après les observa- tions recueillies dans les vergers pilotes du Réseau d'avertissements phytosanitaires du M.A.P.A.Q. depuis 1987 (M. Roy, données non publiées), l'action des ennemis naturels semblerait plutôt marginale dans les vergers conventionnels. Dans les vergers biologiques ou à utilisation minimale de pesticides, on a observé une plus grande variété d'espèces bénéfiques.

\section{MOYENS DE LUTTE ACTUELS}

Actuellement, la stratégie de lutte contre les acariens phytophages repose essentiellement sur l'utilisation d'acaricides et vise à maintenir les populations à un niveau économiquement tolérable jusqu'à la récolte, et ce, au meilleur coût possible (Roy et Vincent 1988).

Les traitements avant la floraison retardent les infestations du tétranyque rouge par la destruction de ses oeufs d'hiver. Des traitements d'huile à demidose au stade phénologique du débourrement avancé du pommier permettent de tuer les oeufs par asphyxie. Si un traitement à l'huile ne peut être effectué, le pomiculteur peut traiter les larves de tétranyque rouge du pommier avec le chinométhionate (6-methyl-1,3-dithioIo[4,5-b]quinoxalin-2-one) au stade du bouton rose ou jusqu'au stade du calice avec la clofentézine [3,6-bis(2-chlorophenyl)-1,2,4,5-tetrazine], un acaricide homologué depuis peu (Roy 1989). Ces deux acaricides permettent de réprimer les larves du tétranyque rouge du pommier, mais ils n'assurent pas toujours une protection durable pour toute la saison. De plus, ils peuvent s'avérer peu efficaces pour la répression des populations de tétranyques à deux points qui migrent sur les pommiers au mois de juin et qui atteignent des niveaux de population élevés à partir de la mi-juillet.

Les traitements d'été sont effectués dès que les populations de tétranyques dépassent les seuils de tolérance. Ces seuils peuvent varier selon la méthode de dépistage utilisée. Une étude récente réalisée au Québec a démontré la possibilité du suivi des populations d'acariens à partir d'un protocole d'échantillonnage 
séquentiel, dont le seuil évolue avec la saison (Binns et Bostanian 1988). Les acaricides recommandés pour les traitements d'été sont le dicofol [2,2,2-tricloro1,1-bis(4-chlorophenyl)ethanol], le chinométhionate et le formétanate (3-dimethylaminomethyleneiminophenyl methylcarbamate).

Les traitements acaricides sont fort coûteux. D'après une enquête réalisée auprès de 55 pomiculteurs québécois (Savoie et al. 1992) ${ }^{2}$, ceux-ci ont effectué en moyenne 2,5 traitements acaricides au cours de la saison 1990. En 1991, les pomiculteurs rattachés au réseau-pommier du M.A.P.A.Q. ont effectué en moyenne 2,3 traitements acaricides. Le coût de ces acaricides s'est élevé en moyenne à $215,45 \$$ ha $^{-1}$, soit $31 \%$ du coût total d'achat de pesticides (Roy et Laplante 1992$)^{3}$.

\section{L'UTILISATION DES ACARIENS PRÉDATEURS EN VERGERS}

Bien que les groupes d'ennemis naturels des acariens phytophages soient nombreux, plusieurs études ont montré le rôle prépondérant des prédateurs de la famille des Phytoséiides, dans la régulation des populations de tétranyques en vergers non traités (Chant 1959; Collyer 1964; Parent 1958; van de Vrie 1962). Pendant une vingtaine d'années, les avis sont demeurés très partagés sur le potentiel d'exploitation des phytoséiides contre les acariens nuisibles en vergers commerciaux (McMurtry 1983).

Au début des années 70 , la découverte aux États-Unis de souches de Metaseiulus occidentalis Nesbitt [Acarina: Phytoseiidae] (Croft et Nelson 1972; Motoyama et al. 1970) et d'Amblyseius fallacis (Ahlstrom et Rock 1973; Croft et Jeppson $1970)$ résistantes à l'azinphos-méthyl [ $S$ (3,4-dihydro-4-oxo-1,2,3-benzotriazin-3ylmethyl) 0,0-dimethyl phosphorodithioate], a ouvert de nouvelles perspectives dans la lutte biologique contre les acariens phytophages en vergers. On rapporte également l'existence de souches de Typhlodromus pyri Sheuten [Acarina: Phytoseiidae] résistantes aux composés organophosphorés aux États-Unis (Watve et Lienk 1976), aux Pays-Bas (Overmeer et van Zon 1983), en Grande-Bretagne (Kapetanakis et Cranham 1983) et en Nouvelle-Zélande (Hoyt 1972). II en est de même de souches d'Amblyseius andersoni Chant [Acarina: Phytoseiidae] signalées en Italie (Gambaro 1975) et en Suisse (Baillod 1986). L'amélioration génétique a par la suite permis le développement en laboratoire de souches résistantes aux pyréthrinoïdes de synthèse chez quelques espèces de phytoséiides dont $A$. fallacis (Strickler et Croft 1982), M. occidentalis (Hoy et Knop 1981) et T. pyri (Markwick 1986). Présentement on connait officiellement une trentaine de souches résistantes sélectionnées naturellement en plein champ ou expérimentalement en laboratoire (Croft 1990). $D$ 'après les études sur la génétique de la résistance aux pesticides, la résistance aux composés organophosphorés ou aux carbamates dépend d'un seul gène, ce qui rend cette résistance très stable $(\mathrm{Croft}$ et al. 1976; Roush et Hoy 1981; Roush et Plapp 1982). La résistance aux pyréthrinoïdes est polygénique, instable et peut se perdre rapidement suite au croisement des populations résistantes avec des populations sensibles (Hoy 1985).

La découverte de souches de phytoséiides résistantes à certaines classes de pesticides a grandement favorisé le développement de programmes de lutte intégrée contre les acariens nuisibles (Croft 1982). Les travaux sur le sujet sont nombreux; Croft (1990) en a fait une revue récente. Les résultats de l'application de ces programmes de lutte ont été très variables. Dans certains cas, on a perçu de nombreux facteurs défavorables, dont le faible niveau de résistance ou l'instabilité de la résistance de la souche utilisée. Par contre des souches de phytoséiides ayant un degré de résistance très élevé ont été introduites et libérées avec succès notamment aux États-Unis (Croft et Barnes 1972; Croft et Meyer 1973; Hoy et al. 1983), en Grande-Bretagne (Aliniazee et Cranham 1980), en Hollande (van de Baan et al. 1985), en URSS (Chubinnishvilli et al. 1982), en Australie (Readshaw 1975), en Nouvelle-Zélande (Penman et al. 1979) et en Chine (Zhang et Kong 1985). 
Au Canada, d'abord en ColombieBritannique, vers la fin des années 60 , on a eu recours au développement d'un programme de lutte intégrée dans les vergers avec l'utilisation de souches de $T$. occidentalis indigènes et résistantes à l'azinphos-méthyl (Downing et Arrand 1976). Dans le sud-ouest de l'Ontario, un inventaire récent des acariens prédateurs dans les pommeraies a permis la découverte d'une souche d'Amblyseius fallacis résistante à la fois aux composés organophosphorés et aux pyréthrinoïdes de synthèse (Thistlewood 1991). La sélection en laboratoire depuis 1988 de cette souche pour la résistance à la perméthrine [3-phenoxybenzyl (1RS)-cis, trans3-(2,2-dichlorovinyl)-2,2-dimethylcyclopropanecarboxylate] a permis d'obtenir un niveau de résistance à cet insecticide très élevé (H. M. A. Thistlewood, communication personnelle). La production de masse de cette souche sur une base commerciale est en cours, ce qui devrait permettre le développement d'un programme de lutte adapté au contexte ontarien. En Nouvelle-Écosse, des études ont montré la présence de $T$. pyri dans les vergers peu traités (Hardman et al. 1991; Rasmy et MacPhee 1970). La sensibilité aux pyréthrinoïdes de ces souches indigènes limite leur utilisation en vergers commerciaux (Hardman et al. 1991). Récemment, on a introduit une souche de $T$. pyri résistante aux pyréthrinoïdes en provenance de Nouvelle-Zélande dans un verger expérimental de la NouvelleÉcosse, pour lutter contre le tétranyque rouge du pommier. L'efficacité de cette souche a été démontrée dans les conditions climatiques de cette province et en présence des pesticides utilisés (Hardman et Bent 1992). L'évaluation de son potentiel d'utilisation en vergers commerciaux en Nouvelle-Écosse se poursuit. (J. M. Hardman, communication personnelle).

\section{LES ESSAIS DE LUTTE BIOLOGIQUE AU QUÉBEC}

Au Québec, un premier essai sur I'utilisation de la lutte biologiquer envers les acariens phytophages a débuté en 1979 et s'est poursuivi pendant 4 ans à la ferme expérimentale d'Agriculture Canada de Frelisghburg (Bostanian et Coulombe
1986). La parcelle traitée comprenait une centaine de pommiers standards de cultivars Mclntosh et Cortland et ne comptait préalablement aucun prédateur indigène. L'essai comportait une souche $\mathrm{d}^{\prime}$ Amblyseius fallacis résistante à certains insecticides organophosphorés provenant de la Station de recherche d'Agriculture Canada à Vineland, Ontario. Après la multiplication de l'acarien en serre sur des plants de haricots, selon la méthode de Rock et Yeargan (1970), un premier lâcher a été effectué en 1979 à raison d'une centaine de prédateurs par arbre. Le rapport proie:prédateur a été étroitement surveillé et des traitements de cyhexatin (tricyclohexylhydroxystannane) à faible dose ont été appliqués au besoin. Les résultats obtenus présentaient beaucoup de variations selon les années. En 1979 et 1980, le prédateur a réduit les populations de tétranyques à un niveau suffisamment bas pour éliminer tout traitement acaricide. En 1981, un hiver très rigoureux et des gelées tardives au printemps ont décimé les populations de prédateurs. En 1982, une application non-intentionelle de bénomyl [methyl 1(butylcarba-moyl)benzimidazol-2-ylcarbamate] élimina toute activité perceptible du prédateur jusqu'en septembre. En 1983, malgré un autre lâcher et des traitements de cyhexatin à dose plus élevée, les prédateurs n'ont pu s'établir et assurer une lutte satisfaisante.

II devint nécessaire d'entreprendre une étude pour la mise au point d'une technique d'élevage de masse et l'évaluation du potentiel d'Amblyseius fallacis en verger commercial. À cette fin, on aménagea en 1991 au Service de phytotechnie de Saint-Hyacinthe, une serre Harnois de fibre de verre dont on divisa une section en trois compartiments et qu'on dota d'un système d'arrosage automatique. Le premier compartiment servit au semis de la plante-hôte, la fève de lima, Phaseolus lunatus L. cv. King, le deuxième compartiment servit à l'élevage du tétranyque à deux points sur cette plante, et le troisième servit à l'élevage du prédateur sur la plante-hôte infestée par le tétranyque à deux points. Des cloisons de plastique et du moustiquaire (Vispore Insect Screen) séparaient les trois compartiments. On disposa des plateaux de culture sur des tables mobiles inondantes. 
Le premier objectif fut d'établir la régie de culture de la plante-hôte. Outre la fève de lima, des tests ont été effectués avec d'autres espèces cultivées: le sorgho soudan (Sorghum sudanense Hitch. Macf. ), le blé (Triticum aestivum L.), I'avoine (Avena sativa L.), le trèfle (Trifolium sp.), la tagète (Tagetes sp.), le maïs (Zea mays L.), le haricot (Phaseolus vulgaris L.), le soja [Glycine max (L.) Merr.] afin de déterminer leur potentiel comme plantehôte du tétranyque à deux points et leur adaptation en serre pendant l'été. De toutes les espèces mises à l'essai, la fève de lima apparut la plus apte à l'élevage de cet acarien. On détermina en second lieu ses exigences d'élevage (température, humidité, stade de la plante-hôte et temps requis pour obtenir une infestation sans mettre la survie de la plante en péril). Les conditions optimales pour la production de la souche d'A. fallacis résistante aux composés organophosphorés et provenant de la Station de recherches de Saint-Jean-sur-Richelieu ont été vérifiées de la même façon. Par des comptages, on a établi le nombre de tétranyques à deux points et de prédateurs par feuille. À une température moyenne de $28^{\circ} \mathrm{C}$ et une humidité relative d'environ $60 \%$, I'obtention de prédateurs avec un rapport prédateur: proie maximal a requis en moyenne $30 \mathrm{j}$. En 1992, on a estimé que la technique d'élevage a permis de produire environ 30000 prédateurs par jour du début juin à la mi-août.

En 1991 à Rougemont et en 1992 à Saint-Paul d'Abbotsford, des lâchers ont été effectués en verger commercial suivant un protocole comprenant les indications sur les périodes et méthodes d'échantillonnage et surtout sur l'emploi d'autres pesticides. Le pomiculteur devait effectuer les traitements antiparasitaires en tenant compte de la toxicité des pesticides sur l'acarien prédateur. Un échantillonnage hebdomadaire devait être conduit afin de déterminer si le seuil de lâcher ( 3 à 5 formes mobiles de tétranyques par feuille) avait été atteint. En 1991, malgré plusieurs lâchers successifs à raison de 100 prédateurs par arbre pendant les mois de juillet et d'août, $A$. fallacis n'a pas réussi à maintenir les populations de tétranyques à un niveau acceptable. Cet échec a été attribué prin- cipalement à la contre-indication expresse d'utiliser le mancozèbe [complexe de sel de zinc de manganèse ethylenebis (dithiocarbamate) (polymérique)], un fongicide très toxique pour le prédateur. Malgré tout, les niveaux de populations d'Amblyseius fallacis en fin de saison pouvaient atteindre une moyenne de 1,7 adultes par feuille. Les comptages ont montré que le prédateur avait pu se reproduire et coloniser les pommiers adjacents aux pommiers inoculés. En 1992, les conditions climatiques ont été défavorables au développement des tétranyques rouges et des tétranyques à deux points et le seuil de lâcher n'a jamais été atteint. On a cependant procédé avec succès à des lâchers à la fin du mois de juillet pour contrer les populations d'ériophyiides du pommier, Aculus Schletendali Nalepa [Acarina: Eriophyidae] qui avaient dépassé le seuil d'intervention de 150 formes mobiles par feuille.

\section{PERSPECTIVES D'AVENIR}

L'utilisation des phytoséiides comme moyen de lutte biologique s'est surtout développée en Europe et aux États-Unis. L'avancement de la recherche dans ce domaine et le développement de programmes de protection où l'on se préoccupait des effets des pesticides sur les prédateurs ont grandement favorisé cet essort.

Au Québec, le recours à la lutte biologique contre les tétranyques en vergers ne suscite qu'un intérêt récent et la recherche sur ce sujet a été amorcée depuis peu. Les programmes de protection constituent une limite importante pour l'utilisation des acariens prédateurs. D'après l'enquête de Savoie et al. (1992) ${ }^{2}$ réalisée sur les pratiques phytosanitaires dans les vergers québécois, six des neuf insecticides les plus utilisés sont très toxiques pour l' $A$. fallacis. Du côté des fongicides, les dithiocarbamates qui sont également toxiques pour les prédateurs, sont les principaux produits utilisés pour réprimer la tavelure.

Dans ce contexte, I'utilisation des phytoséiides résistants aux composés organophosphorés et aux pyréthrinoides est la seule façon d'envisager le développe- 
ment de la lutte biologique contre les tétranyques. Afin d'arriver à élaborer une véritable stratégie de lutte contre ces acariens phytophages, il faut intensifier les efforts de la recherche portant sur les acariens prédateurs. D'abord, on doit réaliser un inventaire des espèces d'acariens prédateurs présentes dans les vergers commerciaux du Québec, pour en évaluer le potentiel et le comparer avec celui des souches d'A. fallacis actuellement disponibles afin de choisir la meilleure espèce prédatrice. Les facteurs principaux à considérer dans le choix d'un acarien prédateur devraient être son adaptation aux conditions climatiques du Québec, son efficacité prédatrice et sa résistance aux pesticides utilisés dans les programmes antiparasitaires.

\section{RÉFÉRENCES}

Ahlstrom, K. P., et G. C. Rock. 1973. Comparative studies on Neoseiulus fallacis and Metaseiulus occidentalis for azinphosmethyl toxicity and effects of prey and pollen on growth. Ann. Entomol. Soc. Am. 66: 1109- 1113.

Aliniazee, M. T. et J. E. Cranham. 1980. Effects of four synthetic pyrethroids on a predatory mite, Typhlodromus pyri and its prey, Panonychus ulmi, on apples in Southeast England. Environ. Entomol. 9: 436-439.

Baillod, M. 1986. Régulation naturelle des tétranyques en vergers de pommiers et perspectives actuelles de lutte biologique à l'aide d'acariens prédateurs phytoséiides. Bull. OILB/SROP IX/3: 5-16.

Binns, M. R. et N. J. Bostanian. 1988. Binomial and censored sampling in estimation and decision making for the negative binomial distribution. Biometrics 44: 473-483.

Bostanian, N. J. et L. J. Coulombe. 1986. An integrated pest management program for apple orchards in southwestern Quebec. Can. Entomol. 118: 1131-1142.

Chant, D. A. 1959. Phytoseiid mites (Acarina: Phytoseiidae). Pt.1 Bionomics of seven species in south-eastern England. Pt. II. A taxonomic review of the family Phytoseiidae, with descriptions of thirty-eight new species. Can. Entomol. 91 (Suppl. 12): 166 pp.

Chubinnishvilli, T. I., Y. V. Koblianidze, A. Z. Petrushov et I. V. Zil'bermints. 1982. [Introduction of resistant Metaseiulus.] (en russe). Zash. Rast. 1: 30-31.
Collyer, E. 1964. A Summary of experiments to demonstrate the role of Typhlodromus pyri Scheut. in the control of Panonychus ulmi (Koch) in England. Acarologia 9: 363371.

Croft, B. A. 1982. Arthropod resistance to insecticides: a key to pest control failures and successes in North American apple orchards. Entomol. Exp. Appl. 31: 88-110.

Croft, B. A. 1990. Arthropod biological control agents and pesticides. John Wiley and Sons, New York. 723 pp.

Croft, B. A. et M. M. Barnes. 1972. Comparative studies on four strains of Typhlodromus occidentalis. VI. Persistence of insecticide resistant strains in an apple orchard ecosystem. J. Econ. Entomol. 65: 211-216.

Croft, B. A. et L. R. Jeppson. 1970. Comparative studies on four strains of Typhlodromus occidentalis. II. Laboratory toxicity to ten compounds common to apple pest control. J. Econ. Entomol. 63: 1528-1531.

Croft, B. A. et R. H. Meyer. 1973. Carbamate and organophosphorous resistance patterns in populations of Amblyseius fallacis. Environ. Entomol. 2: 691-695.

Croft, B. A. et E. E. Nelson. 1972. Toxicity of apple orchard pesticides to Michigan populations of Amblyseius fallacis. Environ. Entomol. 1: 576-579.

Croft, B. A., A. W. A. Brown et S. A. Hoying. 1976. Organophosphorous resistance and its inheritance in the predaceous mite Amblyseius fallacis. J. Econ. Entomol. 69: 64-68.

Downing, R. S. et J. C. Arrand. 1976. Integrated control of orchards mites on apple in British Columbia. Can. Entomol. 108: 77 81.

Flexner, J. L., P. H. Westigard, P. Gonzalves et R. Hilton. 1991. The effect of ground cover and herbicide treatment on twospotted spider mite density and dispersal in southern Oregon pear orchards. Entomol. Exp. Appl. 60: 111-123.

Gambaro, P. I. 1975. Selezione di popolazioni de Acari predatori resistenti ad alcumi insectidi fosfonatiorganici. Estrat. Inform. Fitopath. 25: 21-25.

Hardman, J. M. et E. Bent. 1992. Importation and establishment of insecticide-resistant strains of the phytoseiid predator Typhlodromus pyri in Nova Scotia. Biocontrol News 5: 34-37.

Hardman, J. M., R. E. L. Rogers, J. P. Nyrop et T. Frisch. 1991. Effect of pesticide applications on abundance of European red mite (Acari: Tetranychidae) and Typhlodromus pyri (Acari: Phytoseiidae) in Nova Scotian apple orchards. J. Econ. Entomol. 84: 570 580. 
Hislop, R. G. et R. J. Prokopy. 1981. Integrated management of phytophagous mites in Massachussetts (USA) apple orchards. 2. Influence of pesticides on the predator Amblyseius fallacis (Acarina: Phytoseiidae) under laboratory and field conditions. Prot. Ecol. 3: 157-172.

Hoy, M. A. 1985. Recent advances in genetics and genetic improvement of the Phytoseiidae. Annu. Rev. Entomol. 30: 345-370.

Hoy, M. A. et N. F. Knop. 1981. Selection for genetic analysis of permethrin resistance in Metaseiulus occidentalis : genetic improvement of a biological control agent. Entomol. Exp. Appl. 30: 10-18.

Hoy, M. A. , P. H. Westigard et S. C. Hoyt. 1983. Release and evaluation of laboratory selected, pyrethroid resistant strains of the predatory mite, Typhlodromus occidentalis, (Acarina, Phytoseiidae) in southern Oregon pear orchards and Washington apple Orchards. J. Econ. Entomol. 76: 383388.

Hoyt, S. C. 1972. Resistance to azinphosmethyl of Typhlodromus pyri (Acarina: Phytoseiidae) from New-Zealand. N.Z. J. Sci. 15: 1621.

Kapetanakis, E. G. et J. E. Cranham. 1983. Laboratory evaluation of resistance to pesticides in the phytoseiid predator $T y$ phlodromus pyri from English apple orchards. Ann. Appl. Biol. 103: 389-400.

MAPAQ (ministère de I'Agriculture, des Pêcheries et de l'Alimentation du Québec).1992. Essais de production intégrée en verger de pommiers et de lutte biologique contre les acariens phytophages. Projet 2A2-46440211-0188. 30 pp.

Markwick, N. P. 1986. Detecting variability and selecting for pesticide resistance in two species of phytoseiid mites. Entomophaga 31: 225-236.

McMurtry, J.A. 1983. Phytoseiid predators in orchards system: a classical biological control success. Pages 21-26 in M.A. Hoy, G.L. Cunningham, L.V. Knutson (réds.), Biological control of pests mites. Div. Agric. Nat. Res. Univ. Calif., Berkeley, Spec. Publ. 3304: $185 \mathrm{pp}$.

Motoyama, N., G. C. Rock et W. C. Dauterman. 1970. Organophosphous resistance in an apple orchard population of Typhlodromus (Amblyseius fallacis). J. Econ. Entomol. 63: 1439-1442.

Overmeer, W. P. J. et A. Q. van Zon. 1983. Resistance to parathion in the predaceous mite, Typhlodromus pyri Scheuten (Acarina: Phytoseiidae). Entomophaga 27: $357-$ 364.

Paradis, R. O. 1955. Cycle évolutif du tétranyque à deux points, Tetranychus bimaculatus Harvey (Acari: Tetranychidae), dans le sud-ouest du Québec. Nat. Can. 82: 5-29.
Parent, B. 1958. Efficacité des prédateurs sur le tétranyque rouge du pommier Panony chus ulmi (Koch), dans les vergers du Québec. Ann. Soc. Entomol. Qué. 4: 62-69.

Parent, B. 1967. Population studies of phytophagous mites and predators on apple in southwestern Quebec. Can. Entomol. 99: 771-778.

Parent, B. 1973. Natural population densities of the European red mite on apple in Quebec. Environ. Entomol. 2: 1064-1068.

Parent, B. et A. A. Beaulieu. 1957. Life-history of the European red mite. Can. Entomol. 99: 328-332.

Parent, B. et J. G. Pilon. 1978. Écologie et dynamique des populations naturelles du tétranyque rouge du pommier, Panonychus ulmi (Koch),(Acarina: Tetranychidae), dans le sud-ouest du Québec. Mém. Soc. Entomol. Qué. 5: 106 pp.

Penman, D. R., C. H. Wearing, E. Collyer et W. P. Thomas. 1979. The role of insecticideresistant phytoseiids in integrated mite control in New-Zealand. Pages 59-69 in J.G. Rodriguez (red.), Recent advances in acarology. Academic Press, New-York.

Pfeiffer, D.G. 1986. Effects of field applications of paraquat on densities of Panonychus ulmi (Koch) and Neoseiulus fallacis (Garman). J. Agric. Entomol. 3: 322-325.

Rasmy, A. H. et A. W. MacPhee. 1970. Mites associated with apples in Nova Scotia. Can. Entomol. 102: 172-174.

Readshaw, J. L. 1975. Biological control of orchard mites in Australia with an insecticide-resistant predator. J. Aust. Inst. Agric. Sci. 41: 213-214.

Rock, G. D. et R. R. Yeargan. 1970. Relative toxicity of miticide to the European red mite, the two-spotted spider mite and the predacious mite Neoseiulus (Typhlodromus) fallacis (Family: Phytoseiidae). Down to Earth 26: 1-4.

Rock, G. D. et R. R. Yeargan. 1973. Toxicity of apple orchard herbicides and growth regulating chemicals to Neoseiulus fallacis and twospotted spider mite. J. Econ. Entomol. 66: 1342-1343.

Roush, R.T. et M.A. Hoy. 1981. Selection for insecticide resistance in Metaseiulus occidentalis (Nesbitt) (Acarina: Phytoseiidae). J. Econ. Entomol. 74: 138-141.

Roush, R. T. et F. W. Plapp. 1982. Biochemical genetics of resistance to aryl carbamate insecticides in the predaceous mite Metaseiulus occidentalis . J. Econ. Entomol. 75: 142-147.

Roy, M. 1989. Homologation d'un nouvel acaricide: Apollo 500SC. Avertissement phytosanitaire. Réseau-Pommier. Cornmuniqué no 2. 1er mai: 2 pp.

Roy, M. et C. Vincent 1988. Les principaux acariens phytophages en pomoculture au Québec. L'Horticulteur 3(9): 4-5,8. 
Roy, M. et C. Vincent 1989. Les ennemis des fruits à pépins au Québec en 1988. Rev. Can. Ins. Nuis. Cult. 65: 45-47.

Strickler, K. et B. A. Croft. 1982. Selection for permethrin resistance in the predatory mite Amblyseius fallacis. Entomol. Exp. Appl. 31: $339-345$.

Thistlewood, H. M. A. 1991. A survey of predatory mites in Ontario apple orchards with diverse pesticide programs. Can. Entomol. 123: $1163-1174$.

van de Baan, H. E., L. A. M. Kuijpers, W. P. J. Overmeer et F. J. Oppenoorth. 1985. Organophosphorous and carbamate resistance in the predaceous mite Typhlodromous pyri due to insensitive acetylcholinesterase. Exp. \& Appl. Acarol. 1: 3-10. van de Vrie, M. 1962. Ecology of tetranychid mites and their natural enemies: A review. III. Biology, ecology, and pest status, and host-plant relations of tetranychids. Hilgardia 41: 343-432.

Vincent, C., M. Roy et D. Lebeau. 1990. Les ennemis des fruits à pépins au Québec en 1989. Rev. Can. Ins. Nuis. Cult. 66: 45-47.

Watve, C. et S. E. Lienk. 1976. Toxicity of carbaryl and six organophosphorous insecticides to Amblyseius fallacis and Typhlodromus pyri from New York orchards. Environ. Entomol. 5: 368-370.

Zhang, N. X. et J. A. Kong. 1985. [Responses of Amblyseius fallacis Garman to various relative humidity regimes] (en chinois). Chin. J. Biol. Control 1(3): 6-9. 